\author{
REVISTA MATEMÁTICA de la \\ Universidad Complutense de Madrid \\ Volumen 10, número 1: 1997 \\ http://dx.doi.org/10.5209/rev_REMA.1997.v10.n1.17485
}

\title{
Galois Module Structure of Generalized Jacobians.
}

\author{
G. D. VILLA-SALVADOR and M. RZEDOWSKI-CALDERÓN
}

\begin{abstract}
For a prime number $\ell$ and for a finite Galois $\ell$-extension of function fields $L / K$ over an algebraically closed field of characteristic $p \neq \ell$, it is obtained the Galois module structure of the generalized Jacobian associated to $L, \ell$ and the ramified prime divisors. In the cyclic case an implicit integral representation of the Jacobian is obtained and this representation is compared with the explicit representation.
\end{abstract}

\section{Introduction}

Let $k$ be an algebraically closed field of characteristic $p>0$. Let $\ell$ be a prime different from $p$. Let $L / k$ be a function field of one variable, $G$ be a finite $\ell$-group of $k$-automorphisms of $L$ and $K$ be the fixed field. Then $L / K$ is a finite Galois $\ell$-extension with Galois group $G$. The group $G$ acts naturally on several $Z_{\ell}$-modules defined on $L, Z_{\ell}$ denoting the ring of $\ell$-adic integers. Let $J_{L}$ be the Jacobian variety associated with $L / k$. Then $G$ acts on $J_{L}$ and, by restriction, on $e^{n} J_{L}$, the points of $J_{L}$ of order dividing $\ell^{n}$. Let $J_{L}(\ell)=\lim _{\rightarrow} \ell^{n} J_{L}$. Then $J_{L}(\ell)$ is naturally $G$-isomorphic to $C_{0 L}(\ell)$, the $\ell$-Sylow subgroup of the class group $C_{0 L}$ of divisors of degree 0 in $L$. It is well known that as groups, $C_{0 L}(\ell) \cong R^{2 g_{L}}$, where $g_{L}$ denotes the genus of $L, R=Q_{\ell} / Z_{\ell}$, and $Q_{\ell}$ denotes the field

A.M.S. Classification: 11R58, 20C10, 20C11

Servicio Publicaciones Univ. Complutense. Madrid, 1997. 
of the $\ell$-adic numbers. The dual of $J_{L}(\ell)$ is the Tate module associated with $L$ and $\ell, T_{\ell}(L) \cong \lim _{\leftarrow} \ell J_{L}$

In this paper we are interested in the Galois module structure of the generalized Jacobian $J_{\mathcal{N}}(\ell) \cong C_{0 \mathcal{N}}(\ell)$, where $\mathcal{N}$ is a modulus in $L$ induced by a modulus $\mathcal{M}$ in $K, \mathcal{M}$ containing all the primes in $K$ ramified in $L$.

Section 2 is devoted to the calculation of the cohomology of ${ }_{\ell} C_{0 L}, C_{0 \mathcal{N}}(\ell)$ and $\ell_{\ell} C_{0 \mathcal{N}}$ which will be used in the last section. In Section 3 we consider the integral representation of the generalized Jacobian (Theorem 6). Our main tool is a theorem of Valentini [5] about the number of regular representations appearing in an $F[G]$-module, $F$ a field of characteristic $\ell, G$ a finite $\ell$-group. From the integral representation of the generalized Jacobian, we are able, in the cyclic case, to obtain an implicit integral representation of the usual Jacobian via the dual of Heller's loop operator. This is Theorem 9. Finally, we compare the implicit integral representation of the Tate module with the explicit one obtained in [2] deriving an expresion for the dual of Heller's loop operator (Corollary 10).

In this paper, for a natural number $m, \mathcal{C}_{m}$ will denote a cyclic group of order $m$. For a finite Galois $\ell$-extension $L / K$ of function fields of one variable over $k, r$ will denote the number of primes in $K$ ramified in $L, r \geq 0 ; G_{1}, \cdots, G_{r}$, will denote the ramification groups. Finally, if the ramification indexes of these $r$ ramified primes are $\ell^{n_{1}}, \ldots, \ell^{n_{r}}$, then we will asume $n_{1} \geq n_{2} \geq \ldots \geq n_{r}$.

\section{Cohomology of Jacobians}

In what follows $D_{L}$ will denote the divisors of the field $L, D_{0 L}$ the divisors of degree $0, P_{L}$ the principal divisors and $C_{L}$ the class group of $L$.

We consider the exact sequences of $Z_{\ell}[G]$-modules:

$$
\begin{gathered}
1 \rightarrow k^{*} \rightarrow L^{*} \rightarrow P_{L} \rightarrow 1 \\
1 \rightarrow P_{L} \rightarrow D_{L} \rightarrow C_{L} \rightarrow 1 \\
1 \rightarrow C_{0 L} \rightarrow C_{L} \stackrel{\stackrel{d}{\rightarrow}}{Z} \rightarrow 0 \\
1 \rightarrow_{\ell} C_{0 L} \rightarrow C_{0 L} \stackrel{\ell}{\rightarrow} C_{0 L} \rightarrow 1
\end{gathered}
$$


Here $d$ denotes the degree map and $\ell$ is exponentiation by $\ell$.

For any $G$-module $A$, the Tate i-th cohomology group $\hat{H}^{i}(G, A)$, $i \in Z$ will be denoted by $H^{i}(A)$ and its cardinality by $h^{i}(A)$. We also denote $A^{G}=\{a \in A \mid g a=a$ for all $g \in G\}, N$ the norm (trace) map, that is $N x=\sum_{g \in G} g x$, and ${ }_{N} A$ will denote the kernel of $N$ acting on $A$.

We also denote $I_{G}=\langle g-1 \mid g \in G\rangle \subseteq \boldsymbol{Z}[G] \subseteq \boldsymbol{Z}_{\ell}[G]$.

In [2] we proved:

Proposition 1. Let $L / K$ be a cyclic extension of degree $\ell^{n}$. Then the cohomology groups of $C_{0 L}$ and $C_{L}$ are given by:

i) If $L / K$ is unramified,

$$
\begin{array}{ll}
H^{0}\left(C_{0 L}\right)=\{0\} ; & H^{0}\left(C_{L}\right)=\{0\} ; \\
H^{1}\left(C_{0 L}\right) \cong \mathcal{C}_{\ell^{n}} \oplus \mathcal{C}_{\ell^{n}} ; & H^{1}\left(C_{L}\right) \cong \mathcal{C}_{\ell^{n}}
\end{array}
$$

ii) If $L / K$ is ramified,

$$
\begin{array}{ll}
H^{0}\left(C_{0 L}\right) \cong \bigoplus_{i=3}^{r}\left(\mathcal{C}_{\ell^{n_{i}}}\right) ; & H^{0}\left(C_{L}\right) \cong \bigoplus_{i=2}^{r}\left(\mathcal{C}_{\ell^{n_{i}}}\right) ; \\
H^{1}\left(C_{0 L}\right) \cong \mathcal{C}_{\ell^{n-n_{1}}} \oplus \mathcal{C}_{\ell^{n-n_{1}}} ; & H^{1}\left(C_{L}\right) \cong \mathcal{C}_{\ell^{n-n_{1}}} .
\end{array}
$$

Proposition 2. If $L / K$ is a cyclic extension of degree $\ell^{n}$, then:

$$
\left|N\left(\ell C_{0 L}\right)\right|=\left\{\begin{array}{cl}
\ell^{2 g_{K}-2} & \text { if } L / K \text { is not totally ramified } \\
\ell^{2 g_{K}} & \text { if } L / K \text { is totally ramified. }
\end{array}\right.
$$

Proof. We consider first the case when $L / K$ is unramified. From (4) we obtain:

$$
\mathcal{C}_{\ell^{n}} \oplus \mathcal{C}_{\ell^{n}} \cong H^{1}\left(C_{0 L}\right) \stackrel{\ell}{\rightarrow} H^{1}\left(C_{0 L}\right) \rightarrow H^{0}\left(\ell C_{0 L}\right) \rightarrow H^{0}\left(C_{0 L}\right)=\{0\},
$$

so that

$$
\frac{{ }_{\ell} C_{0 L}^{G}}{N\left({ }_{\ell} C_{0 L}\right)} \cong H^{0}\left({ }_{\ell} C_{0 L}\right) \cong \frac{H^{1}\left(C_{0 L}\right)}{\ell H^{1}\left(C_{0 L}\right)} \cong \mathcal{C}_{\ell}^{2}
$$

Since the extension $L / K$ is unramified, the conorm map $\Psi$ satisfies $\Psi\left(D_{K}\right)=D_{L}^{G}, \Psi\left(D_{0 K}\right)=D_{0 L}^{G}$ and $\Psi\left(C_{0 K}\right) \subseteq C_{0 L}^{G}$. From

$$
1 \rightarrow P_{L} \rightarrow D_{0 L} \rightarrow C_{0 L} \rightarrow 1,
$$


we have

$$
D_{0 L}^{G}=D_{0 K} \rightarrow C_{0 L}^{G} \rightarrow H^{1}\left(P_{L}\right)=\{0\},
$$

and we obtain that $\Psi\left(C_{0 K}\right)=C_{0 L}^{G}$. Since $C_{0 K}$ is divisible, we have $\ell_{\ell} C_{0 K} \cong{ }_{\ell} C_{0 L}^{G}$. Therefore we have

$$
\left|N\left(\ell C_{0 L}^{G}\right)\right|=\ell^{2 g_{K}} .
$$

We also obtain

$$
h^{0}\left({ }_{\ell} C_{0 L}\right)=\left[{ }_{\ell} C_{0 L}^{G}: N\left({ }_{\ell} C_{0 L}\right)\right]=\left[{ }_{\ell} C_{0 K}: N\left({ }_{\ell} C_{0 L}\right)\right]=\ell^{2} .
$$

Thus,

$$
\left|N\left({ }_{\ell} C_{0 L}\right)\right|=\ell^{2 g_{K}-2} .
$$

When $L / K$ is ramified, we obtain from (4) that

$$
\begin{aligned}
\rightarrow H^{1}\left(C_{0 L}\right) \stackrel{\beta}{\rightarrow} & H^{0}\left(\ell C_{0 L}\right) \stackrel{\alpha}{\rightarrow} H^{0}\left(C_{0 L}\right) \stackrel{\ell}{\rightarrow} H^{0}\left(C_{0 L}\right) \rightarrow H^{1}\left(\ell C_{0 L}\right) \rightarrow \\
& \rightarrow H^{1}\left(C_{0 L}\right) \stackrel{\ell}{\rightarrow} H^{1}\left(C_{0 L}\right) \stackrel{\beta}{\rightarrow} H^{0}\left(\widehat{\ell} C_{0 L}\right) .
\end{aligned}
$$

Hence, im $\alpha=\operatorname{ker} \ell=\mathcal{C}_{\ell}^{r-2}\left(\right.$ since $\left(H^{0}\left(C_{0 L}\right) \cong \bigoplus_{i=3}^{\tau}\left(\mathcal{C}_{\ell^{r_{i}}}\right)\right)$ and $\operatorname{im} \beta=\operatorname{ker} \alpha$.

Now, $\operatorname{ker} \beta \cong \operatorname{im}\left[\ell: H^{1}\left(C_{0 L}\right) \rightarrow H^{1}\left(C_{0 L}\right)\right]$ and since

$$
H^{1}\left(C_{0 L}\right) \cong \mathcal{C}_{\ell^{n-n_{1}}} \oplus \mathcal{C}_{\ell^{n-n_{1}}}
$$

we have

$$
\operatorname{ker} \beta=\left\{\begin{array}{lccc}
\mathcal{C}_{\ell^{n-n_{1}}-1} & \oplus & \mathcal{C}_{\ell^{n-n_{1}}-1} & \text { if } n_{1}<n \\
& \{0\} & & \text { if } n_{1}=n
\end{array} .\right.
$$

From $h^{1}\left(C_{0 L}\right)=\ell^{2\left(n-n_{1}\right)}=|\operatorname{im} \beta||\operatorname{ker} \beta|$ it follows that

$$
|\operatorname{ker} \alpha|=|\operatorname{im} \beta|=\left\{\begin{array}{cl}
\ell^{2} & \text { if } n_{1}<n \\
\ell^{2\left(n-n_{1}\right)}=1 & \text { if } n_{1}=n
\end{array} .\right.
$$

Then

$$
h^{0}\left(\ell_{\ell} C_{0 L}\right)=|\operatorname{im} \alpha| \mid \text { ker } \beta \mid=\left\{\begin{array}{cl}
\ell^{r} & \text { if } n_{1}<n \\
\ell^{r-2} & \text { if } n_{1}=n
\end{array} .\right.
$$




\section{Therefore}

$$
H^{0}\left({ }_{\ell} C_{0 L}\right) \cong \frac{\ell C_{0 L}^{G}}{N\left(\ell C_{0 L}\right)} \cong\left\{\begin{array}{cl}
\mathcal{C}_{\ell}^{r} & \text { if } L / K \text { is not totally ramified } \\
\mathcal{C}_{\ell}^{r-2} & \text { if } L / K \text { is totally ramified. }
\end{array}\right.
$$

When $L / K$ is totally ramified, we will prove that $N: \ell_{\ell} C_{0 L} \rightarrow{ }_{\ell} C_{0 K}$ is surjective. In order to show this, it suffices to prove it for $n=1$. So we assume $L / K$ cyclic of order $\ell, N: C_{0 L} \rightarrow C_{0 K}$ is surjective, and if $x \in \ell_{\ell} C_{0 K}$, then $x$ is a norm from $C_{0 L}$. Now by (4),

$$
H^{1}\left(C_{0 L}\right) \stackrel{\ell}{\rightarrow} H^{1}\left(C_{0 L}\right)=\{0\} \stackrel{\beta}{\rightarrow} H^{0}\left(\ell C_{0 L}\right) \stackrel{\alpha}{\rightarrow} H^{0}\left(C_{0 L}\right) \stackrel{\ell}{\rightarrow} H^{0}\left(C_{0 L}\right)
$$

and $\ell$ is the 0 -map in this case, so

$$
\text { ker } \ell=\operatorname{im} \alpha=H^{0}\left(C_{0 L}\right) \text {. }
$$

Therefore

$$
\frac{\ell^{C} G L}{N\left({ }_{\ell} C_{0 L}\right)} \cong H^{0}\left({ }_{\ell} C_{0 L}\right) \cong H^{0}\left(C_{0 L}\right) \cong \frac{C_{0 L}^{G}}{N\left(C_{0 L}\right)}
$$

In the ramified case the conorm map is injective. Thus $x \in C_{0 K} \subseteq$ $C_{O L}^{G}$ so that the class of $x$ in $H^{0}\left(C_{0 L}\right)$ is 0 , then the class of $x$ is 0 in $H^{0}\left({ }_{\ell} C_{0 L}\right)$ and consequently $x \in N\left({ }_{\ell} C_{0 L}\right)$. Therefore $N:{ }_{\ell} C_{0 L} \rightarrow{ }_{\ell} C_{0 K}$ is surjective as claimed.

Hence, when $L / K$ is totally ramified,

$$
H^{0}\left({ }_{\ell} C_{0 L}\right) \cong \frac{\ell C_{0 L}^{G}}{N\left({ }_{\ell} C_{0 L}\right)} \cong \frac{\ell^{C} G}{{ }_{\ell} C_{0 K}} \cong C_{\ell}^{r-2}
$$

and

$$
\left|\ell C_{0 L}^{G}\right|=\ell^{2 g_{K}-2+r}
$$

Now if $L / K$ is not totally ramified, let $T$ be the maximal unramified extension of $K$ in $L$. We have $N\left({ }_{\ell} C_{0 L}\right)=N_{L / K}\left({ }_{\ell} C_{0 L}\right)=$ $N_{T / K}\left(N_{L / T}\left(\ell C_{0 L}\right)\right)=N_{T / K}\left(\ell C_{0 T}\right)$ and since $T / K$ is not ramified, $\left[{ }_{\ell} C_{0 K}: N_{T / K}\left({ }_{\ell} C_{0 T}\right)\right]=\ell^{2}$. Hence,

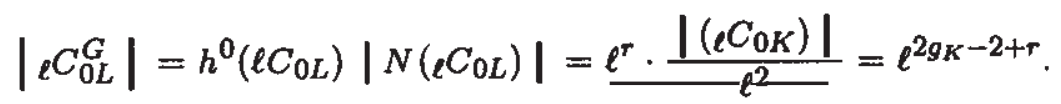


In any case, $\left|\ell_{0 L} C_{0 L}^{G}\right|=\ell^{2 g_{K}-2+\tau}$ and $\left|N\left(\ell C_{0 L}\right)\right|=\frac{\left|\ell C_{0 L}^{G}\right|}{h^{0}\left(\ell C_{0 L}\right)}=\left\{\begin{array}{cl}\ell^{2 g_{K}}-2 & \text { if } L / K \text { is_not_totally_ramified } \\ \ell^{2 g_{K}} & \text { if } L / K \text { is totally ramified. }\end{array}\right.$

Now we consider $L / K$ a Galois $\ell$-extension with Galois group $G,|G|=$ $\ell^{n}$. Let $\wp_{1}, \wp_{2}, \ldots, \wp_{r}, \wp_{r+1}, \ldots, \wp_{r+u}$ be $r+u>0$ different primes in $K$ where $\wp_{1}, \wp_{2}, \cdots, \wp_{r}$ are all the primes ramified in $L$. Let $\mathcal{M}$ be the modulus in $K$ given by $\mathcal{M}=\prod_{i=1}^{r+u} \wp_{i}$ and let $|\mathcal{M}|$ denote the number $r+u$. Let $\mathcal{N}$ be the modulus in $L$ defined by $\mathcal{N}=\prod_{\mathcal{P} \mid p_{i}} \mathcal{P}, 1 \leq i \leq r+u$. If $\ell^{n_{1}}, \cdots, \ell^{n_{r}}$ are the ramification indexes of $\wp_{1}, \wp_{2}, \ldots, \wp_{r}$, we have that $\wp_{i}=\left(\mathcal{P}_{1}^{(i)}, \ldots, \mathcal{P}_{\ell^{n-n_{i}}}^{(i)}\right)^{\ell^{n_{i}}}, 1 \leq i \leq r+u$ (of course $n_{i}=0$ for $r+1 \leq i \leq r+u$ ) and since the ramification is tame, the different is given by

$$
\mathcal{D}_{L / K}=\prod_{i=1}^{\Gamma}\left(\prod_{j=1}^{\ell^{n-n_{i}}} \mathcal{P}_{j}^{(i)^{\ell^{n_{i}}-1}}\right)
$$

The Genus Formula gives us:

$$
\begin{gathered}
2 g_{L}-2=|G|\left(2 g_{K}-2\right)+\operatorname{deg}\left(\mathcal{D}_{L / K}\right)= \\
=|G|\left(2 g_{K}-2\right)+\sum_{i=1}^{\tau} \ell^{n-n_{i}}\left(\ell^{n_{i}}-1\right) .
\end{gathered}
$$

We will use the following notation:

$D_{\mathcal{N}}=$ Divisors in $L$ relatively prime to $\mathcal{N} \subseteq D_{L}$,

$D_{0 \mathcal{N}}=D_{\mathcal{N}} \cap D_{0 L}$

$P_{\mathcal{N}}=\left\{(\alpha) \mid \alpha \in L^{*}, \alpha \equiv 1 \bmod \mathcal{N}\right\}$

$C_{\mathcal{N}}=D_{\mathcal{N}} / P_{\mathcal{N}}$

$C_{0 \mathcal{N}}=D_{0 \mathcal{N}} / P_{\mathcal{N}}$

$L_{\mathcal{N}}=\left\{\alpha \in L^{*} \mid(\alpha)\right.$ is relatively prime to $\left.\mathcal{N}\right\}$,

$L_{\mathcal{N}^{1}}=\left\{\alpha \in L_{\mathcal{N}} \mid \alpha \equiv 1 \bmod \mathcal{N}\right\}$,

$T_{\mathcal{N}}=\{(\alpha) \mid(\alpha)$ is relatively prime to $\mathcal{N}\}$,

and a similar one for $\mathcal{M}$. The generalized Jacobian is $J_{\mathcal{N}} \cong C_{0 N}$. 
Proposition 3. Let $L / K$ be any finite Galois $\ell$-extension and $\mathcal{M} a$ modulus in $K$ containing all the ramified primes. Then $\left|N\left({ }_{\ell} C_{0 \mathcal{N}}\right)\right|=$ $\ell^{2 g_{K}-1+|\mathcal{M}|-d}$ where $d$ is the minimum number of generators of $G$.

Proof. We have the $G$-exact sequences:

$$
\begin{gathered}
1 \rightarrow k^{*} \cap L_{\mathcal{N}^{1}} \rightarrow L_{\mathcal{N}^{1}} \rightarrow P_{\mathcal{N}} \rightarrow 1 \\
1 \rightarrow P_{\mathcal{N}} \rightarrow D_{0 \mathcal{N}} \rightarrow C_{0 \mathcal{N}} \rightarrow 1 \\
1 \rightarrow P_{\mathcal{N}} \rightarrow D_{\mathcal{N}} \rightarrow C_{\mathcal{N}} \rightarrow 1 \\
1 \rightarrow C_{0 \mathcal{N}} \rightarrow C_{\mathcal{N}} \stackrel{d}{\rightarrow} \boldsymbol{Z} \rightarrow 0
\end{gathered}
$$

We have $k^{*} \cap L_{\mathcal{N}^{1}}=\{1\}$. So $L_{\mathcal{N}^{1}} \cong P_{\mathcal{N}}$. The natural maps $\pi: D_{\mathcal{N}} \rightarrow$ $C_{L}$ and $\pi_{0}: D_{0 \mathcal{N}} \rightarrow C_{0 L}$ are surjective and $\operatorname{ker} \pi=$ ker $\pi_{0}=T_{\mathcal{N}}$ so that $C_{L} \cong D_{\mathcal{N}} / T_{\mathcal{N}}$ and $C_{0 L} \cong D_{0 \mathcal{N}} / T_{\mathcal{N}}$. Therefore we obtain the exact sequences of $G$-modules:

$$
\begin{aligned}
1 \rightarrow T_{\mathcal{N}} / P_{\mathcal{N}} & \rightarrow C_{\mathcal{N}} \rightarrow C_{L} \rightarrow 1 \\
1 \rightarrow T_{\mathcal{N}} / P_{\mathcal{N}} & \rightarrow C_{0 \mathcal{N}} \rightarrow C_{0 L} \rightarrow 1
\end{aligned}
$$

We also have that as $Z_{\ell}[G]$-module

$$
\left(T_{\mathcal{N}} / P_{\mathcal{N}}\right)(\ell) \cong \frac{\bigoplus_{i=1}^{r} R\left[G / G_{i}\right] \oplus R[G]^{u}}{R e^{*}},
$$

where $R e^{*}$ denotes the diagonal copy of $R$ in $\bigoplus_{i=1}^{r} R\left[G / G_{i}\right] \oplus R[G]^{u}$ (see $[3],[6]$ and [7]).

Therefore we have the $G$-exact sequence:

$$
0 \rightarrow \frac{\bigoplus_{i=1}^{r} R\left[G / G_{i}\right] \oplus R[G]^{u}}{R e^{*}} \rightarrow C_{0 \mathcal{N}}(\ell) \rightarrow C_{0 L}(\ell) \rightarrow 1
$$

Note that as groups, $C_{0 \mathcal{N}}(\ell) \cong R^{\lambda_{\mathcal{N}}}$ where $\lambda_{\mathcal{N}}=2 g_{L}+|\mathcal{N}|-1$.

By Hilbert's Theorem 90, $H^{1}\left(L_{\mathcal{N}^{1}}\right)=\{0\}$. We also have $P_{\mathcal{N}}^{G} \cong$ $P_{\mathcal{M}}$ and $N P_{\mathcal{N}}=P_{\mathcal{M}}$ so $H^{0}\left(P_{\mathcal{N}}\right)=\{0\}$. Therefore $P_{\mathcal{N}}$ and $L_{\mathcal{N}^{1}}$ are cohomologically trivial. Also $H^{0}\left(D_{\mathcal{N}}\right)=H^{1}\left(D_{\mathcal{N}}\right)=\{0\}$. Therefore, from $(7), C_{\mathcal{N}}$ is cohomologically trivial. 
From $(8) H^{i}(Z) \cong H^{i+1}\left(C_{0 N}\right)$. Therefore $H^{0}\left(C_{0 \mathcal{N}}\right) \cong H^{-1}(Z)$ $=\{0\}, H^{1}\left(C_{0 \mathcal{N}}\right) \cong H^{0}(Z) \cong Z /|G| Z$ and $H^{-1}\left(C_{Q \mathcal{N}}\right) \cong H^{-2}(Z) \cong$ $G / G^{\prime}$

Now we consider the $G$-exact sequence

$$
1 \rightarrow \ell C_{\mathrm{QN}} \rightarrow C_{\mathrm{QN}} \stackrel{\ell}{\rightarrow} C_{0 N} \rightarrow 1
$$

From (11),

$$
H^{-1}\left(C_{0 N}\right) \stackrel{\ell}{\rightarrow} H^{-1}\left(C_{Q N}\right) \rightarrow H^{0}\left({ }_{\ell} C_{Q N}\right)=H^{0}\left(C_{Q N}\right)=\{0\}
$$

Therefore $H^{0}\left({ }_{\ell} C_{0 \mathcal{N}}\right) \cong \frac{H^{-1}\left(C_{0 N}\right)}{\ell H^{-1}\left(C_{\mathrm{aN}}\right)} \cong G / G^{\ell} G^{\prime}=G / \Phi(G) \cong \mathcal{C}_{\ell}^{d}$, where $\Phi(G)$ is the Frattini subgroup of $G$ and $d$ is the minimum number of generators of $G$.

Now the conorm map $\varphi: C_{0 M} \rightarrow C_{Q \mathcal{N}}^{G}$ is surjective (see [6, pag 266, Proposition 9]). Therefore

$$
\left|\ell_{0 N} C^{G}\right|=\ell^{\lambda M}=\ell^{2 g_{K}-1+|\mathcal{M}|}
$$

Since $H^{0}\left({ }_{\ell} C_{0 \mathcal{N}}\right) \cong \frac{\ell C_{0 N}^{C}}{N\left(\ell C_{0 N}\right)} \cong \mathcal{C}_{\ell}^{d}$, it follows that

$$
\left|N\left({ }_{\ell} C_{0 \mathcal{N}}\right)\right|=\ell^{2 g_{K}-1+|\mathcal{M}|-d} \text {. }
$$

\section{Integral Representations}

First we recall a theorem of Valentini [5]. He states this result when $F$ is an algebraically closed field, but it is valjd for a general field $F$ of characteristic $\ell$.

Proposition 4. Let $F$ be a field of characteristic $\ell, G$ be a finite $\ell$-group and $M$ be a finitely generated $F[G]$-module. Let $N$ be the norm map and $n=\operatorname{dim}_{F} N(M)$. Then $M \cong F[G]^{n} \oplus P$, where $F[G]$ is not a component of $P$. 
Let $M$ be a $Z_{\ell}[G]$-module, $G$ a finite $\ell$-group such that $M$ is $Z_{\ell^{-}}$ injective and as a group, $M \cong R^{s}, s<\infty$. Let $\ell M$ be the kernel of multiplication by $\ell$ on $M$. Then $\ell M$ is a finitely generated $F_{\ell}[G]$-module. The proof of the following proposition is given in [2]:

Proposition 5. Let $M$ and $G$ be as above. If $\ell^{M} \cong F_{\ell}[G]^{u} \oplus U$, with $F_{\ell}[G]$ not a component of $U$ and $M \cong R[G]^{v} \oplus V$, with $R[G]$ not a component of $V$, then $u=v$.

From Propositions 3, 4 and 5 we will obtain the structure of the generalized Jacobian for a general $\ell$-group $G$ and also the implicit structure of the usual Jacobian in the cyclic ramified case.

Let $\wp_{1}, \wp_{2}, \ldots, \wp_{r}, \wp_{r+1}, \ldots, \wp_{r+u}$ be a set of primes of $K$, the first $r$ being the ramified primes in the finite Galois $\ell$-extension $L / K, r \geq 0$ and $r+u>0$. Let $G=G$ al $(L / K)$. Let $\mathcal{M}$ be the modulus in $K$ given by $\mathcal{M}=\prod_{i=1}^{r+u} p_{i}$ and let $\mathcal{N}=\prod_{\mathcal{P} \mid}^{r+u} \mathcal{P}$ be the modulus induced by $\mathcal{M}$ in $L$.

By Proposition 3, $\operatorname{dim}_{F}\left(N\left({ }_{\ell} C_{0 N}\right)\right)=2 g_{K}+|\mathcal{M}|-1-d$, where $d$ is the minimum number of generators of $G$. By Proposition $4, \ell C_{0 \mathcal{N}} \cong \boldsymbol{F}_{\ell}[G]^{2 g_{K}+|\mathcal{M}|-1-d} \oplus U$, with $\boldsymbol{F}_{\ell}[G]$ not a component of $U$. Finally, by Proposition 5, we have that $J_{\mathcal{N}}(\ell) \cong R[G]^{2 g_{K}+|\mathcal{M}|-1-d} \oplus S$, with $R[G]$ not a component of $S$.

Theorem 6. With the notation as above, the $\boldsymbol{Z}_{\ell}[G]$-module structure of the generalized Jacobian is given by $J_{\mathcal{N}}(\ell) \cong R[G]^{2 g_{K}+|\mathcal{M}|-1-d} \oplus S$, with $S$ an indecomposable $Z_{\ell}[G]$-module such that, as groups, $S \cong R^{s}$, where $s=|G|(d-1)+1$.

Proof. On the one hand, $\lambda_{\mathcal{N}}=|G|\left(2 g_{K}+|\mathcal{M}|-1-d\right)+s$. On the other, if $\ell^{n_{1}}, \ell^{n_{2}}, \ldots, \ell^{n_{r}}$ are the ramification indexes of $\wp_{1}, \wp_{2}, \ldots, \wp_{r}$, since $\lambda_{\mathcal{N}}=2 g_{L}+|\mathcal{N}|-1$, we obtain from the Genus Formula:

$$
\begin{aligned}
& \lambda_{\mathcal{N}}=2 g_{L}+|\mathcal{N}|-1= \\
& =|G|\left(2 g_{K}-2\right)+2+\sum_{i=1}^{r} \ell^{n-n_{i}}\left(\ell^{n_{i}}-1\right)+|\mathcal{N}|-1= \\
& =|G|\left(2 g_{K}-2\right)+2+|G| \sum_{i=1}^{r}\left(1-\frac{1}{\ell^{n_{i}}}\right)+\sum_{i=1}^{r} \ell^{n-n_{i}}+|G| u-1=
\end{aligned}
$$


$=|G|\left(2 g_{K}-2+r+u\right)+1=|G|\left(2 g_{K}-2+|\mathcal{M}|\right)+1$.

Hence $s=|G|(d-1)+1$.

Now, if $S$ were decomposable, say $S=A \oplus B$, we would have $H^{i}\left(C_{0 \mathcal{N}}\right) \cong H^{i}(S) \cong H^{i}(A) \oplus H^{i}(B)$. We have $H^{0}\left(C_{O N}\right) \cong H^{-1}(Z)=$ $\{0\}$, therefore $H^{0}(A)=H^{0}(B)=\{0\}$ and $H^{1}\left(C_{0 \mathcal{N}}\right) \cong H^{0}(Z) \cong$ $Z /|G| Z \cong H^{1}(A) \oplus H^{1}(B)$, hence $H^{1}(A)=\{0\}$ or $H^{1}(B)=\{0\}$. Therefore $A$ or $B$ would be cohomologically trivial and $Z_{\ell}$-divisible so it would have $R[G]$ as a component, contrary to our assumptions.

Corollary 7. If $G$ is cyclic, then $C_{0 \mathcal{N}}(\ell) \cong R[G]^{2 g_{K}-2+|\mathcal{M}|} \oplus R$.

Proof. In this case, $d=1$.

We finish by analysing the cyclic ramified case. Let $L / K, \mathcal{M}, \mathcal{N}$ as before, $G$ cyclic of order $\ell^{n}$. We assume $r>0, u=0$. Again we denote by $G_{1}, \ldots, G_{r}$ the ramification groups.

For any subgroup $H$ of $G$, if we let $G / H$ denote the left cosets, we call $I_{G, H}$ the indecomposable module

$$
\left\{\sum_{g \in G} a_{g} g \in Z_{\ell}[G] \mid \sum_{g \in G} a_{g}=0 \text { for all } \sigma \in G / H\right\} \text {. }
$$

The proof of the following theorem is given in [2]:

Theorem 8. For any cyclic extension $L / K$ of degree $\ell^{n}$ with Galois group $G$, we have that

$$
T_{\ell}(L)=Z_{\ell}[G]^{2 g_{K}-2} \oplus T_{m+t, t}^{2} \oplus\left(\bigoplus_{i=3}^{r} I_{G, G_{i}}\right)
$$

as $Z_{\ell}[G]$-modules, where $\ell^{m}$ is the degree of the maximal unramified extension of $K$ in $L$, and $T_{m+t, t}$ is an indecomposable module of $Z_{\ell}$ $\operatorname{rank} \ell^{m+t}-\ell^{m}+1$. 
Theorem 9. Let $L / K$ be a finite cyclic ramified $\ell$-extension with Galois group $G, \wp_{1}, \ldots, \wp_{r}$ be the primes in $K$ ramified in $L$ and let $G_{1}, \ldots, G_{r}$ be the corresponding decomposition subgroups. If $\rho$ is given as below, then the $Z_{\ell}[G]$-module structure of the Jacobian $J_{L}(\ell)$ of $L$ is given by $J_{L}(\ell) \cong R[G]^{a} \oplus \Omega^{\#}($ ker $\rho)$ with $R[G]$ not a component of $\Omega^{\#}($ ker $\rho)$, $a=\left\{\begin{array}{ll}2 g_{K}-2 & \text { if } L / K \text { is not totally ramified } \\ 2 g_{K} & \text { if } L / K \text { is totally ramified }\end{array}\right.$, and $\Omega^{\#}$ the dual of Heller's loop operator.

Proof. In this case (10) becomes

$$
0 \rightarrow \frac{\bigoplus_{i=1}^{r} R\left[G / G_{i}\right]}{R e^{*}} \stackrel{i}{\rightarrow} C_{0 \mathcal{N}}(\ell) \stackrel{\pi}{\rightarrow} C_{0 L}(\ell) \rightarrow 1
$$

and by Corollary $7,(12)$ reduces to

$$
0 \rightarrow \frac{\bigoplus_{i=1}^{r} R\left[G / G_{i}\right]}{R e^{*}} \stackrel{i}{\rightarrow} R[G]^{2 g_{K}-2+r} \oplus R \stackrel{\pi}{\rightarrow} C_{0 L}(\ell) \rightarrow 1
$$

Let $f: R[G] \rightarrow R$ be given by $f\left(\sum_{g \in G} a_{g} g\right)=\sum_{g \in G} a_{g}$.

Then we have a $\boldsymbol{Z}_{\ell}[G]$-epimorphism

$$
\theta=(\mathrm{id}, \mathrm{f}): R[G]^{2 g_{K}-1+r}=R[G]^{2 g_{K}-2+r} \oplus R[G] \rightarrow R[G]^{2 g_{K}-2+r} \oplus R .
$$

Thus $\rho=\pi \circ \theta: R[G]^{2 g_{K}-1+r} \rightarrow C_{0 L}(\ell)$ is a $Z_{\ell}[G]$-epimorphism.

$$
\stackrel{r}{\oplus} R\left[G / G_{i}\right]
$$

Let $\mathcal{R}=\frac{{ }_{i=1}}{R e^{*}}$ and $P=R[G]^{2 g_{K}-1+\tau}$. Then we have the commutative diagram

$$
\begin{gathered}
0 \rightarrow \mathcal{R} \stackrel{i}{\rightarrow} C_{0 \mathcal{N}}(\ell) \stackrel{\pi}{\rightarrow} C_{0 L}(\ell) \rightarrow 0 \\
\theta \uparrow \nearrow_{\rho} \\
0 \rightarrow \operatorname{ker} \rho \stackrel{j}{\rightarrow} \quad \mathrm{P}
\end{gathered}
$$

By the dual of Schanuel's Lemma ([6], [7]), this diagram characterizes $C_{0 L}(\ell)$ as $Z_{\ell}[G]$-module. 
Now ker $\rho$ is the pull back of $i$ and $\theta$ ([1]) and hence ker $\rho \cong\{(a, b) \in \mathcal{R} \oplus \mathrm{P} \mid i(a)=\theta(b)\}$.

By Propositions 2, 4 and 5, we obtain that $C_{0 L}(\ell) \cong R[G]^{a} \oplus M$

with $a=\left\{\begin{array}{lll}2 g_{K}-2 & \text { if } L / K \text { is not totally ramified } \\ 2 g_{K} & \text { if } L / K \text { is totally ramified }\end{array}\right.$ and $R[G]$

is not a component of $M$.

Using the dual of Heller's loop operator $\Omega^{\#}([6])$ we obtain that

$$
C_{0 L}(\ell) \cong R[G]^{a} \oplus \Omega^{\#}(\text { ker })
$$

We have that the dual of $I_{G, G_{i}}$ is isomorphic to $\frac{R[G]}{R\left[G / G_{i}\right]}$ Therefore, if $S_{m+t, t}$ denotes the dual of $T_{m+t, t}$, comparing Theorems 8 and 9 we obtain:

Corollary 10. Under the conditions of Theorem 9 we have

$$
\Omega^{\#}(\operatorname{ker} \rho) \cong\left\{\begin{array}{cl}
S_{m+t, t}^{2} \oplus & \left(\bigoplus_{i=3}^{r} \frac{R[G]}{R\left[G / G_{i}\right]}\right) \\
& \text { if } L / K \text { is not totally ramified } \\
\bigoplus_{i=3}^{r} \frac{R[G]}{R\left[G / G_{i}\right]} & \text { if } L / K \text { is totally ramified }
\end{array}\right.
$$

\section{References}

[1] Hilton, Peter J. and Stammbach, Urs, A Course in Homological Algebra , Graduate Texts in Math. 4, Berlin-Heidelberg-New York, (1971).

[2] Rzedowski-Calderón, Martha, Villa-Salvador, Gabriel D. and Madan, Manohar L., "Galois Module Structure of Tate Modules", Math. Z. (to appear). 
[3] Serre, Jean-Pierre, "Algebraic Groups and Class Fields", Graduate Texts in Math. 117, Berlin-Heidelberg-New York, (1988).

[4] Serre, Jean-Pierre, "Local Fields", Graduate Texts in Math. 67, Berlin-Heidelberg-New York, (1979).

[5] Valentini, Robert C., "Representations of automorphisms on differentials of function fields of characteristic $p$, J. Reine und Angew. Math. 335, (1982), 164-179.

[6] Villa-Salvador, Gabriel D. and Madan, Manohar L., "Integral representations of p-class groups in $Z_{p}$-extensions, semisimple differentials and Jacobians", Arch. Math. 56, (1991), 254-269.

[7] Villa-Salvador, Gabriel D. and Madan, Manohar L., "Structure of Semisimple Differentials and p-Class Groups in $Z_{p}$-extensions", Manuscripta Math. 57, (1987), 315-350.

Departamento de Matemáticas

Centro de Investigación y

de Estudios Avanzados del I.P.N.

A partado Postal 14-740

07000 México, D.F.

Recibido: 5 de Diciembre de 1995

MEXICO

Revisado: 17 de Junio de 1996

Gabriel D.Villa Salvador

e-mail:villa@math. cinvestav.mx

Martha Rzedowski Calderón

e-mail:mrzedows Omath. cinvestav.mx 